\title{
Radiation Dose Burden To The Thyroid Gland Following Postoperative Radiotherapy Of Breast Cancer: A Comparative Dosimetric Study
}

\author{
Amr Sakr, Khaled Thabet, Wael S. Makar and Mohammed El-Sayed \\ Kasr El-Aini Center of Oncology and Nuclear Medicine (NEMROCK), Faculty of Medicine, Cairo \\ university, Egypt
}

Purpose: To compare and evaluate Thyroid gland radiation doses in intensity modulated radiotherapy (IMRT) and 3D-conformal radiotherapy (3D-CRT) technique for postoperative treatment of breast cancer.

Patients and methods: Twenty patients with breast cancer, IMRT and 3D-CRT plans were generated using XIO planning system to analyze and compare with respect to doses to thyroid gland using the parameters (Dmax, Dmin, Dmean, and V5Gy).

Results: Thyroid radiation doses were more in IMRT than in 3D-conformal technique particularly Dmax in (Breast conservative surgery) BCS cases which showed statistical significance $(833.60 \pm 38.14 \mathrm{cGy}$ Vs $113.6 \pm 12.93 \mathrm{cGy}, P$-value $<0.05)$. V5Gy was statistically significant in all cases $(7.70 \pm 1.43 \% \mathrm{Vs} 0.00 \%$, with $P$-value $=0.0001$ ).

Conclusion: IMRT associated with excess radiation doses to thyroid gland than the 3D-conformal technique in postoperative radiotherapy of breast cancer.

Key words: IMRT, 3D-conformal radiotherapy, breast cancer, thyroid gland.

Corresponding Author: Wael S. Makar

E-mail: wael_makar@yahoo.com

\section{INTRODUCTION}

The rationale behind adjuvant post-operative radiotherapy following breast-conserving surgery (BCS) is the sterilization of the tumor bed of any residual subclinical disease that may be present after surgical excision. Adjuvant radiotherapy has been shown to be effective in reducing the risk of local recurrence in early stage disease and some studies also demonstrate improved survival in high-risk premenopausal women. The late widespread adoption of BCS and adjuvant post-operative radiotherapy, especially in good prognosis young women with early stage breast disease, increased the importance of late complications due to the long expected disease-free interval ${ }^{1}$.

Second primary malignancies (SPMs) occurring after oncological treatment have become a major concern during the past decade. Their incidence has long been underestimated because most patients had a short life expectancy after treatment or their follow-up was shorter than 15 years. With major improvement of longterm survival, longer follow-up, cancer registries and end-result programs, it was found that the cumulative incidence of SPM could be as high as $20 \%$ of patients treated by radiotherapy ${ }^{2}$.
Population- and hospital-based studies suggested that among breast cancer survivors, the risk of developing a second cancer at other sites is $10-40 \%$ higher than in the general population ${ }^{3,4}$.

The risk of cancer following ionising radiation has been extensively studied and is relatively well known compared to the risks due to other carcinogens ${ }^{5}$. However, little data are available for breast cancer patients even though radiation therapy is widely used to reduce the risk of local recurrence. The relative risk of second malignant neoplasm (SMN) associated with external radiotherapy is between 0.7 and $1.8^{6,7}$.

The conditions in radiotherapy units delivering high doses to limited volumes are quite different from those in the cohorts studied to estimate the risk of ionizing radiation. Consequently, predicting the risks of radiation for breast cancer requires an estimate of the relationship between the radiation dose at a given site and the risk of SMN at this site. Owing to the heterogeneity of the distribution of the radiation dose through the body, the overall role of radiotherapy in SMN risk can only be directly investigated by studying this relationship for all the sites of SMN together ${ }^{5}$. 


\section{PATIENT AND METHODS}

Twenty patients with Stage I, II or III breast who underwent breast conservative surgery (BCS) or modified radical mastectomy (MRM), Level I-II lymph node dissection, and locoregional RT at Kasr El-Aini cancer center (NEMROCK), were chosen for this study. For each patient 3D-CRT and IMRT plans were generated.

\section{Target delineation}

The thyroid gland was delineated by a senior radiation oncologist and with the help of the radiologist. All visible gland was included in all the CT scan cuts as shown in Figures $(1,2)$.

\section{Treatment Planning}

A 3-D CT scan with 5-mm slice spacing was performed. A radiation oncologist delineated the planning target volume (PTV). The PTV comprised the left breast and the chest wall in cases of breast conservative surgery (BCS), and the left chest wall in cases of modified radical mastectomy (MRM). The Internal mammary chain (IMC) PTV was defined by an elliptical cylinder, with a major (lateral) and minor (anterior-posterior) axes of 30 and $20 \mathrm{~mm}$, respectively, centered on the IMC vessels. This extended between the inferior aspect of the ipsilateral clavicular head and the fourth intercostals space to ensure only the first three intercostals spaces were included. The volume of the contralateral breast, the lung and the heart were defined as organs at risk (OARs). The isocenter was positioned in the middle of the PTV.

In 3D-CRTplans, The PWTF plans were performed using standard forward planning methods, the gantry angle was optimized in the beam's eye view (BEV) for a minimum lung area and beam divergence toward the lung was compensated by adjusting the gantry angle of the beams. The ipsilateral lung was spared using a multileaf collimator (MLC). The shape of the MLC was defined in the BEV with a distance of $10 \mathrm{~mm}$ to the PTV to compensate the penumbra in craniocaudal direction and toward the lung Figures $(3,4)$.

In IMRT plans, Seven co-planer equi-angular beams are to be used Figure (5). The treatment planning system generates the beam intensity profiles with a bixel (or beam element) size of $5 \times 5 \mathrm{~mm}^{2}$, using step and shoot IMRT. Dose calculation will be via pencil-beam method. Cost functions are selected and determined to satisfy the plan goals regarding the target coverage and risk organs protection. Optimization uses superposition algorithm. All beam weights and intensity profiles are optimized using Helios inverse planning IMRT module. Optimization is performed by means of a steepest gradient search algorithm, then the segmentation process accomplished according to leaf motion calculator (LMC) algorithm. The maximum number of iterations will be 1000 . Dose constrains to PTV $\&$ organs at risk are estimated numerically and also using constrains, and the optimization process is started and online modifications could be attempted during optimization process to be able to get the best calculated fluence map and dose distribution. Then the segmentation process starts to build the actual fluence for each beam according to leaf constrains of the treatment machine and the process accomplished via LMC algorithm. All plans were calculated at the XIO version 4.2 planning system. Photon energy of 6 MV Elekta accelerator was used.

Dose-volume histograms (DVHs) were generated for all relevant structures for both techniques. Specific metrics were chosen for comparison of the IMRT and 3D-CRT plans Figures (6,7). Dmax, Dmin, Dmean, and V5Gy were compared.

\section{Statistical Analysis}

All statistical calculations were done using computer package SPSS version 16 (statistical package for the social science; SPSS Inc., Chicago, IL, USA) statistical program for Microsoft widows.

\section{RESULTS}

Four parameters were used to evaluate the radiation dose to thyroid gland (Dmax, Dmin, Dmean and V5Gy). All of these parameters were numerically more in IMRT than in 3D-conformal technique, but V5Gy showed statistical significance in the IMRT technique as shown in Table (1).

In the subgroup of patients who had breast conservative surgery, the parameters used to evaluate radiation dose to thyroid gland (Dmax, Dmin, Dmean and V5Gy) were all numerically more in the IMRT than in 3D-conformal technique. Dmax and V5Gy were also statistically significant in the IMRT technique as shown in Table (2).

In the subgroup of patients who had modified radical mastectomy, the parameters used to evaluate radiation dose to thyroid gland (Dmax, Dmin, Dmean and V5Gy) were all numerically more in the IMRT than in 3D-conformal technique. V5Gy was statistically significant in the IMRT technique as shown in Table (3). 
Kasr-El-Aini Journal Of Clinical Oncology And Nuclear Medicine

Vol. 8 | No. 1-2 $2012 \quad$ Wael S. Makar et al.

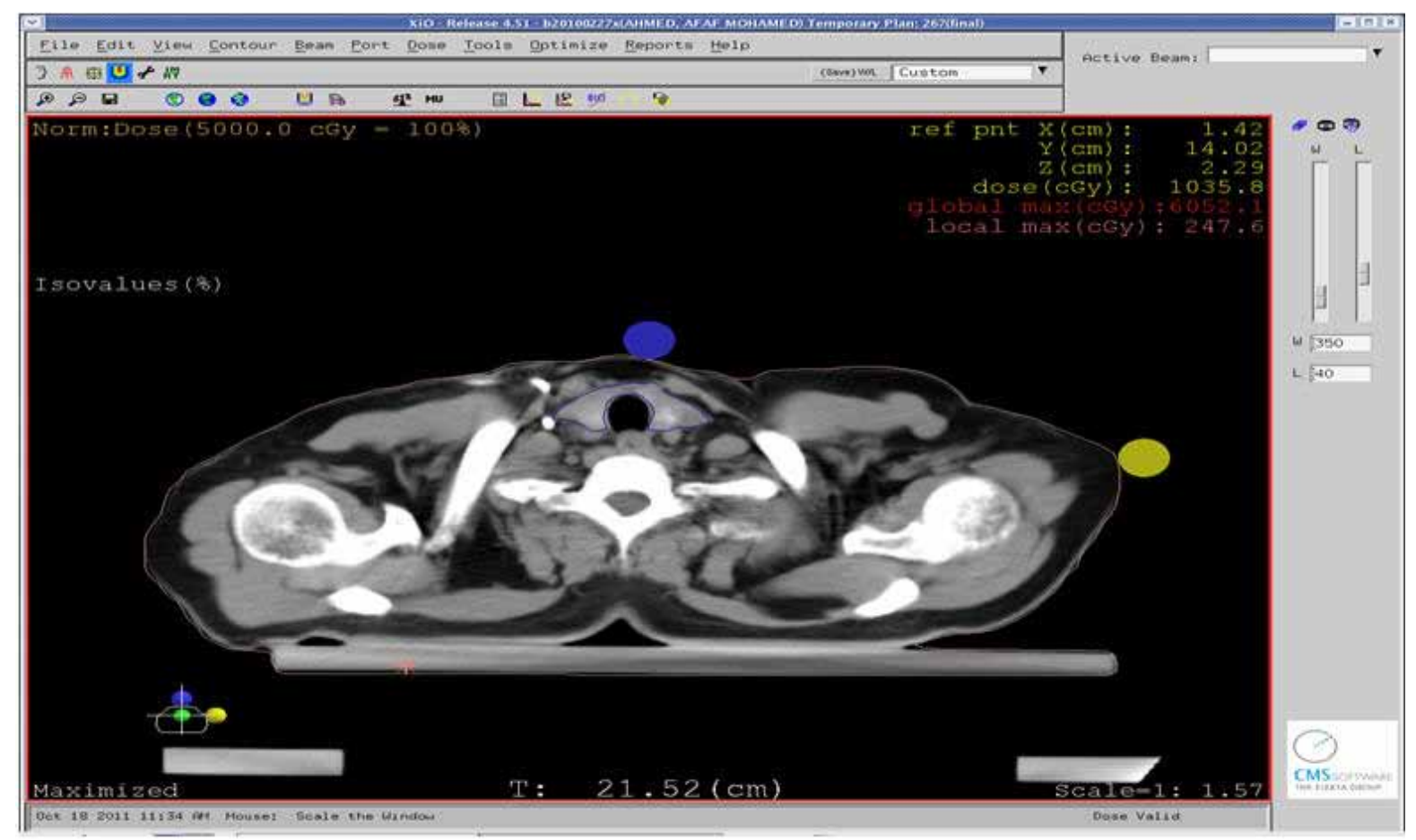

Figure 1: Showed the delineation of the thyroid gland on CT planning.

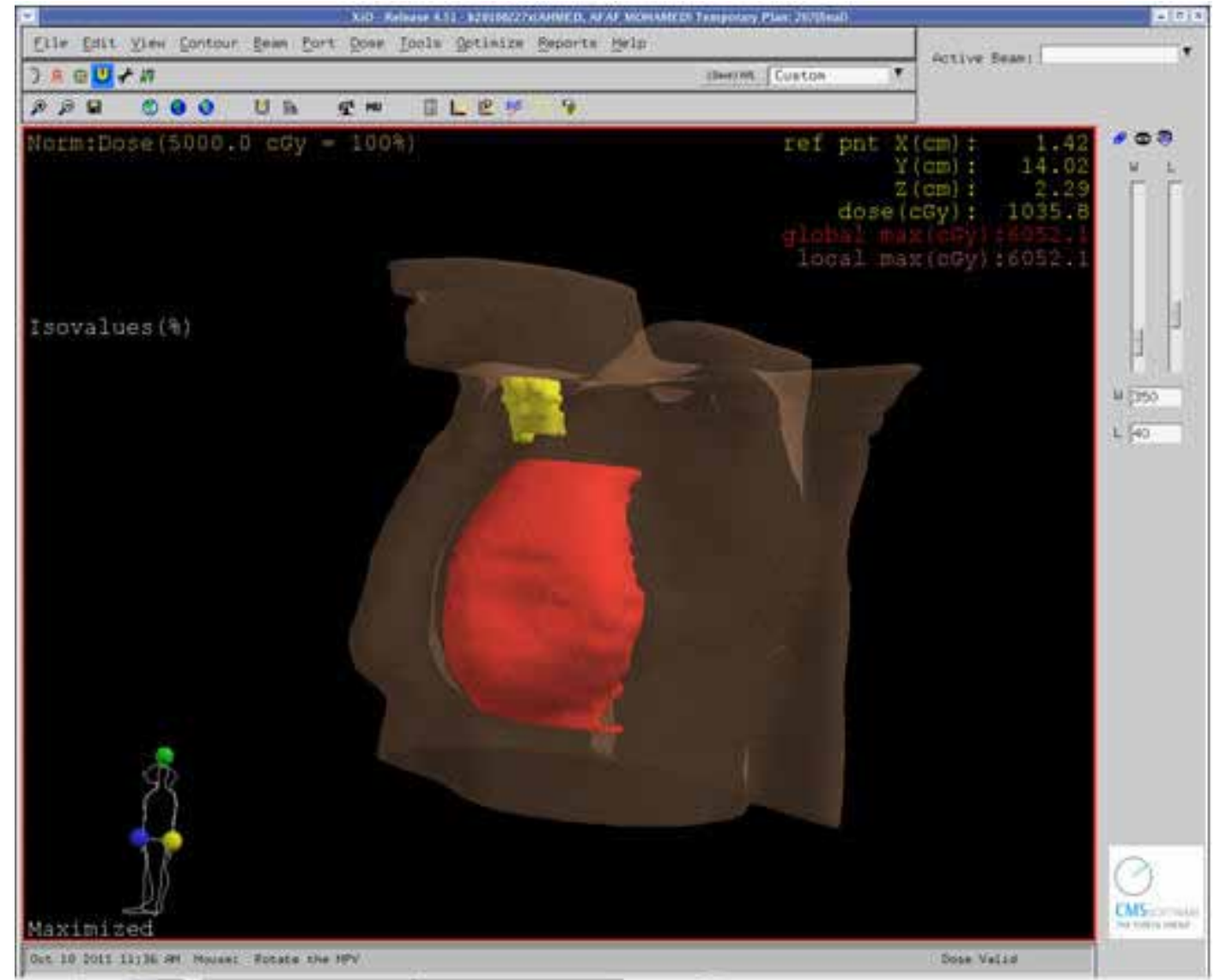

Figure 2: Showed the three-dimensional relation of thyroid gland to the PTV (breast after BCS). 
Kasr-El-Aini Journal Of Clinical Oncology And Nuclear Medicine

Vol. 8 | No. 1-2 $2012 \quad$ Radiation Dose Burden To The Thyroid Gland Following Postoperative Radiotherapy Of Breast Cancer

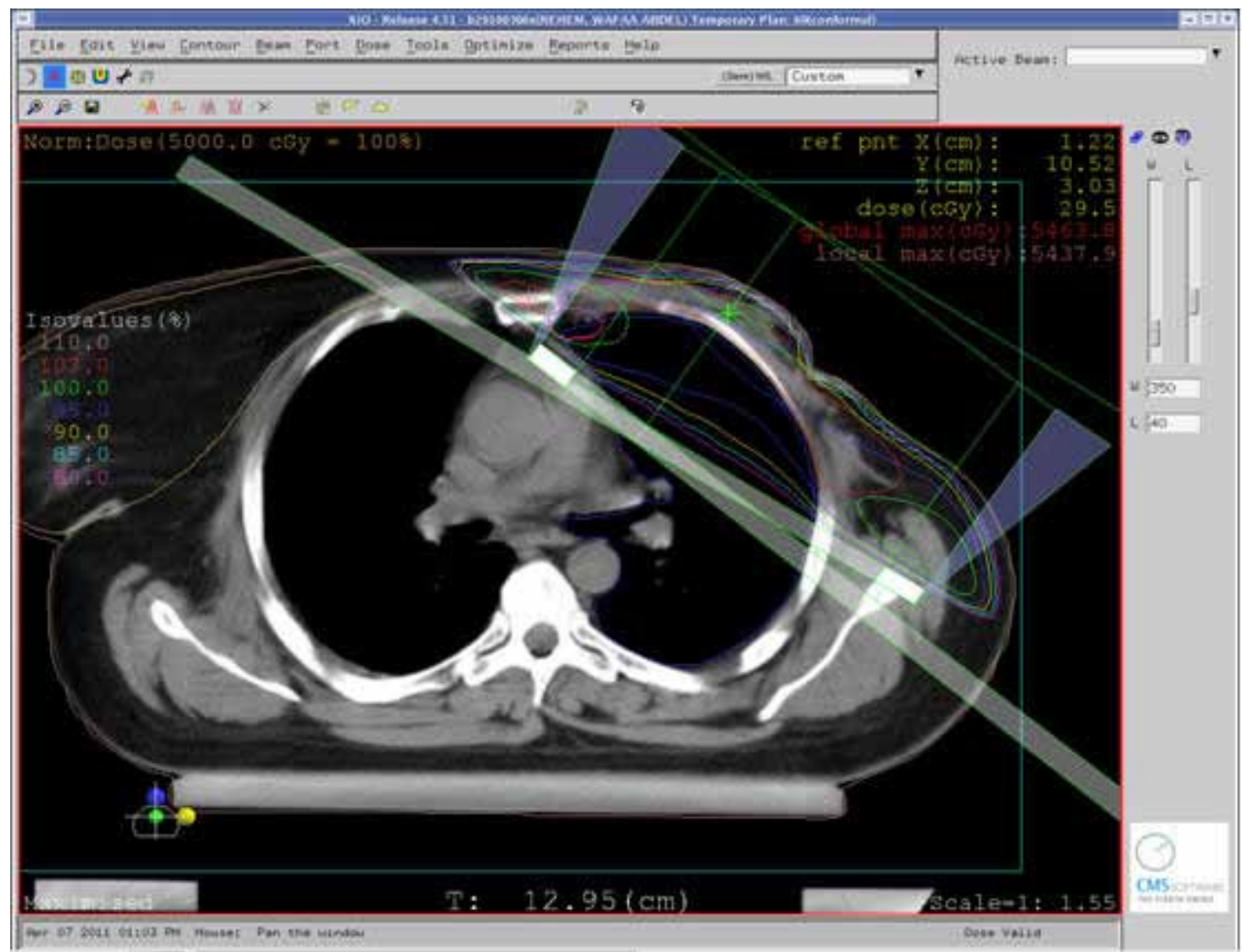

Figure 3: Shows the 3DCRT planning with the two PWTF.

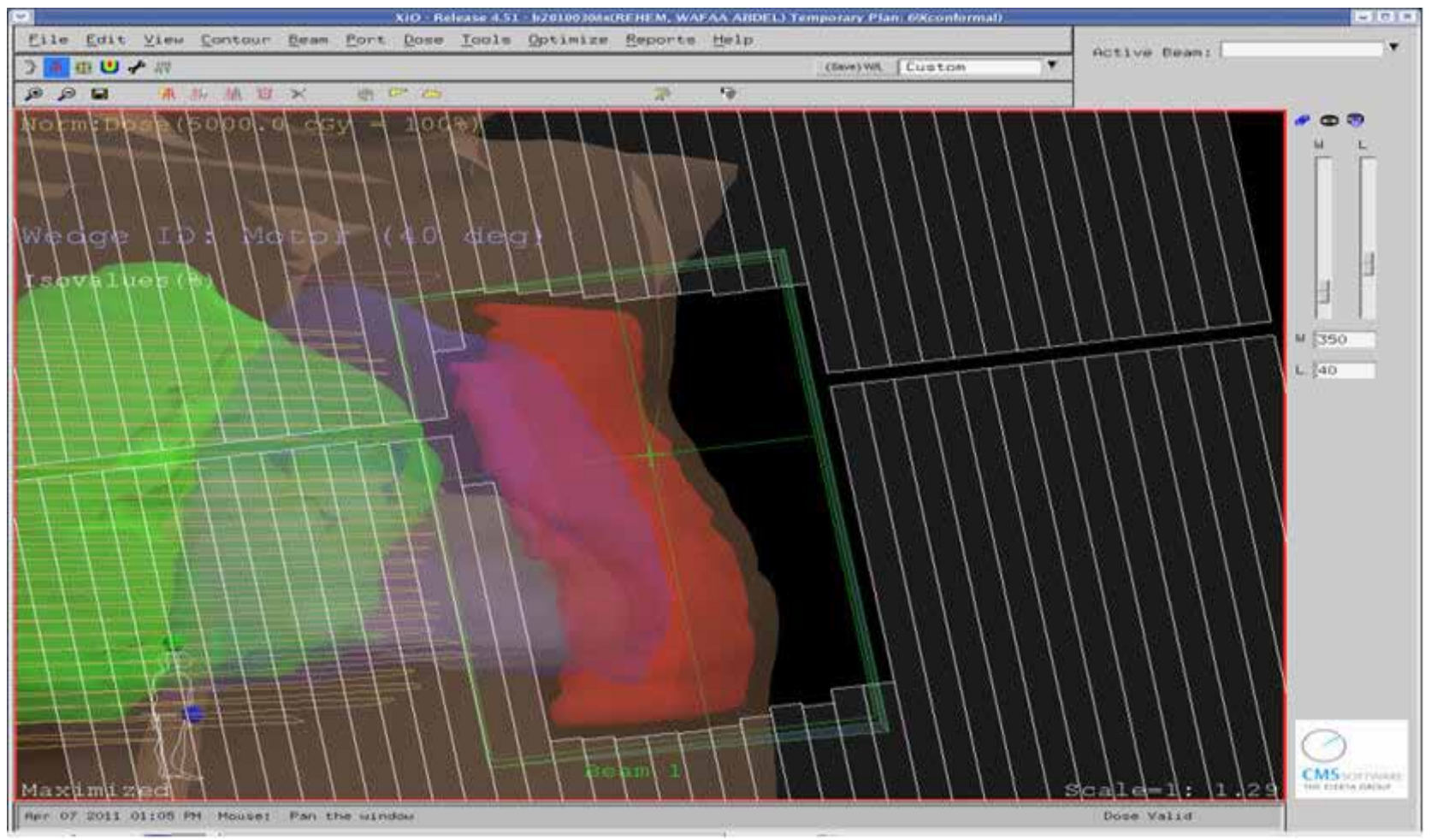

Figure 4: Shows the BEV for the 3d-CRT plan. 
Kasr-El-Aini Journal Of Clinical Oncology And Nuclear Medicine

Vol. 8 | No. 1-2 $2012 \quad$ Wael S. Makar et al.

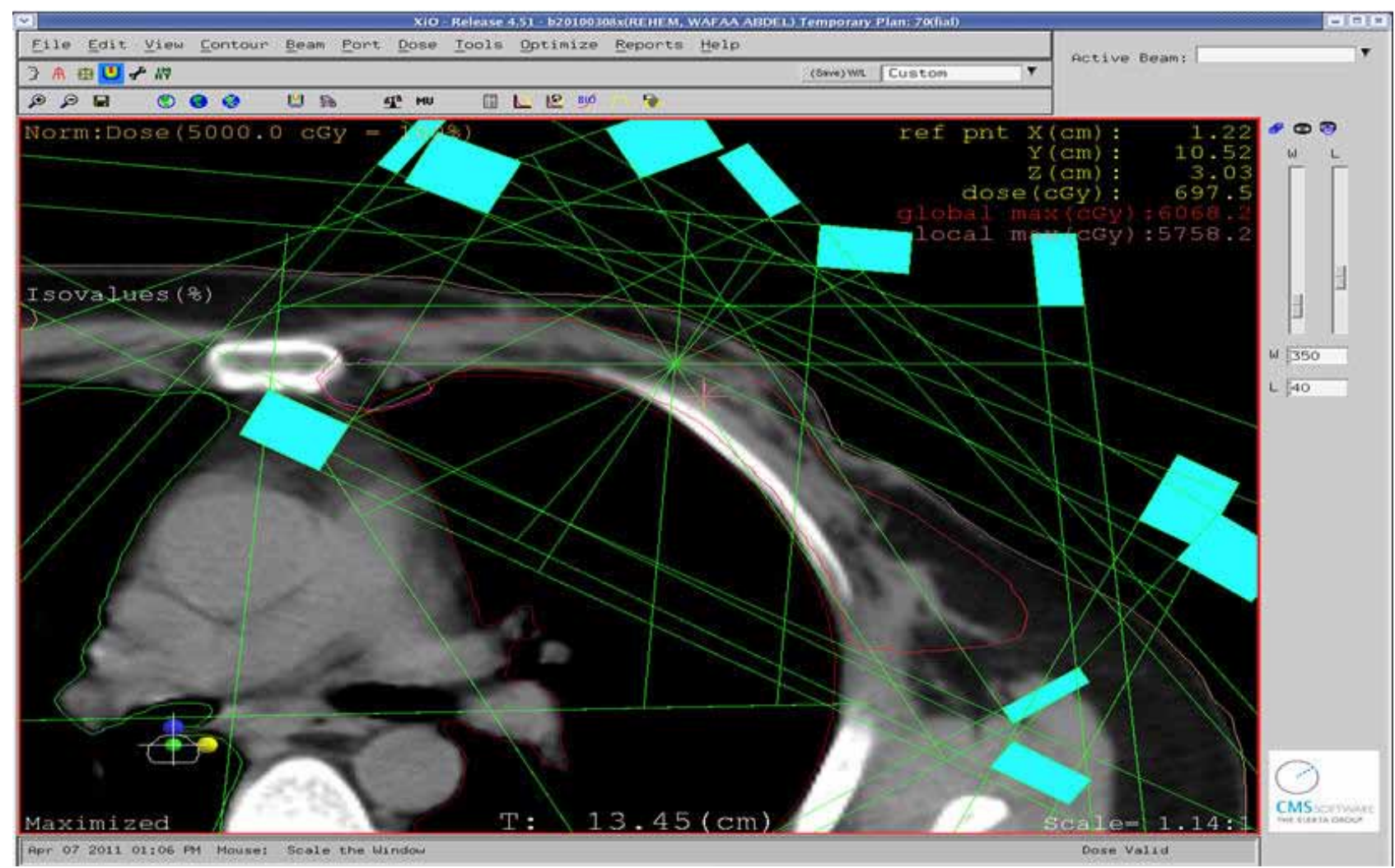

Figure 5: Shows the seven equi-distant co-planner beams in IMRT planning.

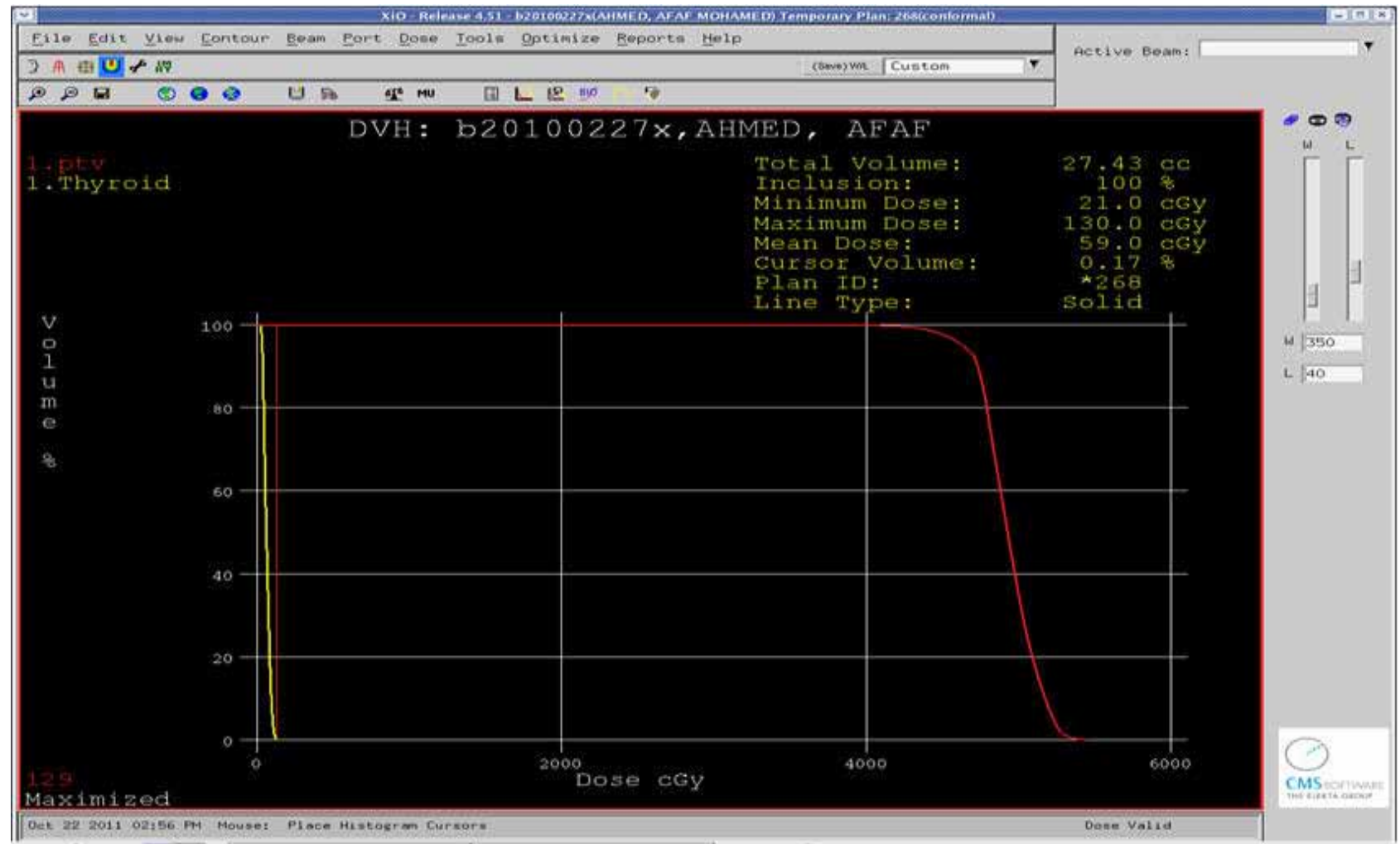

Figure 6: Showed the DVH for the 3D-conformal plan with doses to the PTV and Thyroid gland. 
Kasr-El-Aini Journal Of Clinical Oncology And Nuclear Medicine

Vol. 8 | No. 1-2 $2012 \quad$ Radiation Dose Burden To The Thyroid Gland Following Postoperative Radiotherapy Of Breast Cancer

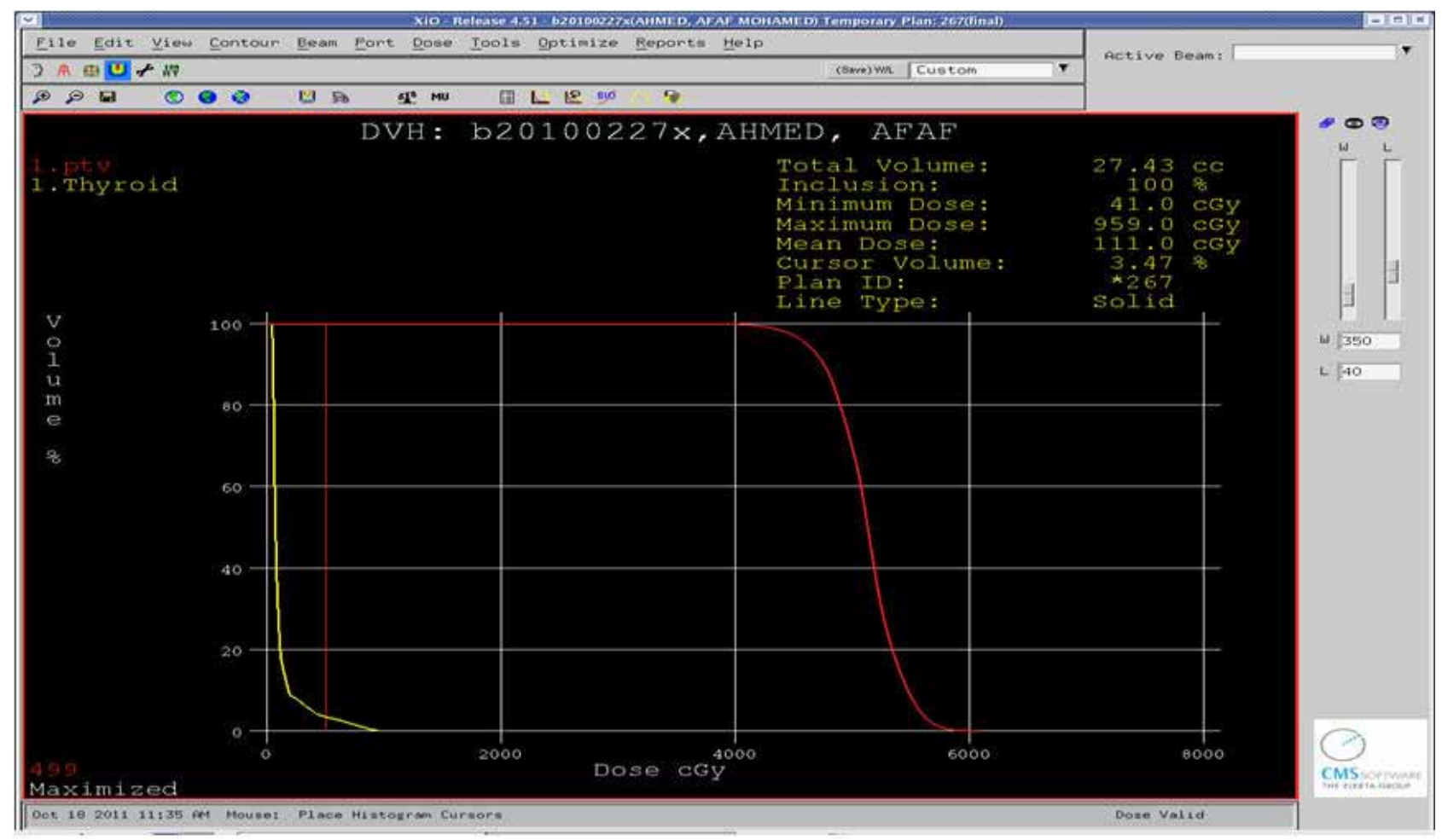

Figure 7: Showed the DVH for the IMRT plan with doses to the PTV and Thyroid gland.

Table 1: Evaluation of Thyroid Radiation Dose all cases.

\begin{tabular}{lccc}
\hline Parameter & $\begin{array}{c}\text { 3D-CRT (20plans) } \\
\text { (mean } \pm \text { Std) }\end{array}$ & $\begin{array}{c}\text { IMRT (20 plans) } \\
\text { (mean } \pm \text { Std) }\end{array}$ & P-Value \\
\hline Dmax & $129.30 \pm 31.46 \mathrm{cGy}$ & $626.0 \pm 238.97 \mathrm{cGy}$ & 0.242 \\
\hline Dmin & $16.20 \pm 5.89 \mathrm{cGy}$ & $39.70 \pm 15.81 \mathrm{cGy}$ & 0.225 \\
\hline Dmean & $55.20 \pm 11.67 \mathrm{cGy}$ & $118.10 \pm 41.53 \mathrm{cGy}$ & 0.254 \\
\hline V5Gy & $0.00 \%$ & $4.85 \pm 1.35 \%$ & 0.0001 \\
\hline
\end{tabular}

Dmax $=$ Dose received by $1 \%$ of thyroid gland

Dmin $=$ Dose received by $99 \%$ of thyroid gland

Dmean $=$ The mean dose received by thyroid gland

$\mathrm{V} 5 \mathrm{~Gy}=$ The percentage of thyroid gland received at least $5 \mathrm{~Gy}$.

Table 2: Evaluation of Thyroid Radiation Dose in BCS (Breast Conservative Surgery) cases.

\begin{tabular}{|c|c|c|c|}
\hline Parameter & $\begin{array}{l}\text { 3D-CRT (10plans) } \\
\quad(\text { mean } \pm \text { Std) }\end{array}$ & $\begin{array}{l}\text { IMRT (10 plans) } \\
\quad(\text { mean } \pm \text { Std })\end{array}$ & $P$-Value \\
\hline Dmax & $113.6 \pm 12.93 \mathrm{cGy}$ & $833.60 \pm 38.14 \mathrm{cGy}$ & 0.046 \\
\hline Dmin & $12.4 \pm 4.3 \mathrm{cGy}$ & $41.20 \pm 19.25 \mathrm{cGy}$ & 0.220 \\
\hline Dmean & $46.0 \pm 10.19 \mathrm{cGy}$ & $151.60 \pm 28.93 \mathrm{cGy}$ & 0.200 \\
\hline V5Gy & $0.00 \%$ & $7.70 \pm 1.43 \%$ & 0.0001 \\
\hline
\end{tabular}


Kasr-El-Aini Journal Of Clinical Oncology And Nuclear Medicine

Vol. 8|No. 1-2 $2012 \quad$ Wael S. Makar et al.

Table 3: Evaluation of Thyroid Radiation Dose in MRM (Modified Radical Mastectomy) cases.

\begin{tabular}{lccc}
\hline Parameter & $\begin{array}{c}\text { 3D-CRT (10 plans) } \\
\text { (mean } \pm \text { Std) }\end{array}$ & $\begin{array}{c}\text { IMRT (10 plans) } \\
\text { (mean } \pm \text { Std) }\end{array}$ & P-Value \\
\hline Dmax & $145.00 \pm 38.0 \mathrm{cGy}$ & $422.40 \pm 146.17 \mathrm{cGy}$ & 0.210 \\
\hline Dmin & $20.00 \pm 13.54 \mathrm{cGy}$ & $38.20 \pm 13.64 \mathrm{cGy}$ & 0.220 \\
\hline Dmean & $64.40 \pm 27.22 \mathrm{cGy}$ & $84.60 \pm 15.42 \mathrm{cGy}$ & 0.520 \\
\hline V5Gy & $0.00 \%$ & $1.99 \pm 0.58 \%$ & 0.0001 \\
\hline
\end{tabular}

\section{DISCUSSION}

The parameters used to evaluate radiation dose to thyroid gland were more in the IMRT than 3D-conformal technique, which means that thyroid gland was exposed to more radiation doses in the IMRT technique. V5Gy was statistically significant in all patient groups. Dmax was only significant in the subgroup of patients who had breast conservative surgery which may be related to the volume of the PTV and the increased scatter dose to thyroid gland. Mansur et al. ${ }^{8}$ showed that The IMRT delivery resulted in higher doses to the more distant points form the PTV, presumably due to the higher monitor units and resulting increased head leakage. The observed doses of radiation to thyroid gland is much less than that which was expected to produce thyroid function abnormalities were the maximum dose which we found didn't exceed 10Gy. Johansen et al. ${ }^{9}$ pointed that the development of hypothyroidism in these patients would primarily depend on the volume receiving relatively high radiation doses ( $\geq 30 \mathrm{~Gy}$ ) thus with the risk of insufficient post-radiotherapy hormone production. This dose was much less than what we found in our study. The thyroid radiation dose was of concern regarding the risk of secondary malignancy. Despite we found that the radiation dose didn't exceed the $10 \mathrm{~Gy}$, but still there was a risk of secondary malignancy. Tubiana, $2009^{2}$ pointed that Preliminary data suggest that second primary malignancies (SPMs) were mainly observed in tissues having absorbed doses above 2 Gy (fractionated irradiation) and that their incidence increases with the dose. However, in children thyroid and breast cancers are observed following doses as low as $100 \mathrm{cGy}$. Ron et al. ${ }^{10}$ showed that the linearexponential model appears to better capture the substantial increased risk for persons treated with more than 2 Gy compared with those treated with less than $2 \mathrm{~Gy}$, and the apparent flattening of the ERR at the very high dose levels. They also found that the pooled excess relative risk per Gy (ERR/Gy) was 7.7 (95\% CI = 2.1, 28.7). These data suggested that the radiation dose to the thyroid gland which we found carried the potential risk for thyroid cancer.

\section{CONCLUSION}

The IMRT technique was shown to increase significantly thyroid radiation doses. This drawback of IMRT was more in breast conservative surgery than modified radical mastectomy cases. Despite the radiation doses felled below the level associated with thyroid functional abnormalities, but still in the range associated with the excess risk of thyroid cancer.

\section{REFERENCES}

1. Cho BCJ, Schwarz M, Mijnheer BJ, Bartelink H. Simplified intensity-modulated radiotherapy using predefined segments to reduce cardiac complications in leftsided breast cancer. Radiother.Oncol. 2004;70(3):231-41.

2. Tubiana M. Can we reduce the incidence of second primary malignancies occurring after radiotherapy? A critical review. Radiother.Oncol. 2009;91(1):4-15.

3. Harvey EB, Brinton LA. Second cancer following cancer of the breast in Connecticut, 1935-82. Natl.Cancer Inst. Monogr. 1985;68:99-112.

4. Rubino C, De Vathaire F, Diallo I, Shamsaldin A, Lê MG. Increased risk of second cancers following breast cancer: Role of the initial treatment. Breast Cancer Res.Treat. 2000;61(3):183-95.

5. UNSCEAR. Sources and effects of ionizing radiation. 2000. United Nations, New York, USA.

6. Valagussa P, Moliterni A, Terenziani M, Zambetti M, Bonadonna G. Second malignancies following CMFbased adjuvant chemotherapy in resectable breast cancer. Ann.Oncol. 1994;5(9):803-8.

7. Tanaka H, Tsukuma H, Koyama H, Kinoshita Y, Kinoshita $\mathrm{N}$, Oshima A. Second primary cancers following breast cancer in the Japanese female population. Japan.J.Cancer Res. 2001;92(1):1-8.

8. Mansur DB, Klein EE, Maserang BP. Measured peripheral dose in pediatric radiation therapy: A comparison of intensity-modulated and conformal techniques. Radiother. Oncol. 2007;82(2):179-84.

9. Johansen S, Reinertsen KV, Knutstad K, Olsen DR, Fosså $\mathrm{SD}$. Dose distribution in the thyroid gland following radiation therapy of breast cancer-a retrospective study. Radiat.Oncol. 2011;6(1):Art. No. 68.

10. Ron E, Lubin JH, Shore RE, Mabuchi K, Modan B, Pottern LM, et al. Thyroid cancer after exposure to external radiation: A pooled analysis of seven studies. Radiat.Res. 1995;141(3):259-77. 